\section{Managing Discovery Risks - A Tevatron Case Study}

\author{
Bakul Banerjee \\ Fermi National Accelerator Laboratory \\ POB 500, Batavia, Illinois 60510 \\ Fermilab-Conf-04/128-CD
}

\section{Abstract}

To meet the increasing need for higher performance, Management of Fermi National Accelerator Laboratory has undertaken various projects to improve systems associated with the Tevatron high-energy particle collider located at Batavia, Illinois. One of the larger projects is the Tevatron Beam Position Monitor (BPM) system. The objective of this project is to replace the existing BPM electronics and software system that was originally installed during early 1980s, along with the original construction of the Tevatron.

The original system consists of 236 beam position monitors located around the underground tunnel of the accelerator. Above ground control systems are attached to these monitors using pickup cables. When the Tevatron collider is operational, signals received from the BPMs are used to perform a number of control and diagnostic tasks. The original system can only capture the proton signals from the collider. The new system, when fully operational, will be able to capture combined proton and antiproton signals and will be able to separate the antiproton signal from the combined signal at high resolution. This significant enhancement was beyond the range of technical capabilities when the Tevatron was constructed about two decades ago. To take advantage of exceptional progress made in the hardware and software area in past two decades, Department of Energy approved funding of the BPM electronics and software replacement project. The approximate length of the project is sixteen months with a budget of four million dollars not including overhead, escalation, and contingencies.

Apart from cost and schedule risks, there are two major risks associated with this research and development project. The primary risk is the risk of discovery. Since the Tevatron beam path is highly complex, BPMs have to acquire and process a large amount of data. In this environment, analysis of data to separate antiproton signals is even more complex. Finding an optimum algorithm that can be implemented with current state of the art hardware and software technology is even more complex. The second most important risk is the risk of unavailability of the premiere high energy physics scientific facility to worldwide users. This paper presents a model of minimizing these risks using a phased model of project management. To complete the project successfully, it is essential to keep track of the constraints imposed by uncertainties in the discovery phase while maintaining the highest possible availability of the Tevatron.

In this paper, a methodology based on earned value management system is explained to measure and manage discovery risks. Metrics based on the initial basis of estimate and familiar earned value measures are used to monitor risks of discovery. Subsequently, these metrics are used to refine the project and adjust tasks and resource assignments to minimize the unavailability of the user facility. One of the measures is the variance at phase completion. From the phased model of the WBS, variance at completion is examined for various project baselines at the end of project phases. This allowed for understanding the risk of not only cost and schedule, but also the discovery risks. Since similar risk measurement data is not available within the organization, these metrics will also allow us to define risk baselines for future scientific projects that involve discovery along with state of the art system development.

\section{Introduction}

In last forty years, small scale high-energy physics faculties around the world transformed into large facilities. The Tevatron collider complex facility at Fermilab, located at Batavia, Illinois was one of the first large scientific user facilities when it was commissioned in early eighties. It will remain the most important facility for high-energy physicists until the commissioning of the Lepton Hadron Collider facility, currently being built at Geneva, Switzerland. However, the new facility will not be useable to scientists until the year 2007. In the mean time, the scientific community desperately needs much higher performance from the existing Tevatron. One of the major components of this project is upgrade of the capabilities of the Tevatron Beam Position Monitoring system.

From the standard project management point of view, this may be perceived as a legacy system upgrade. However, the challenge turned out to be much more than that. The planned system upgrade included major enhancements in general functionalities of the system and new signal processing schemes to increase the capabilities of the Tevatron.

\section{Technical description of the project}

One of the main purposes of the Fermilab's accelerator complex is to produce large amount of collisions among 
protons and antiprotons traveling in opposite directions. These collisions occur in the Tevatron accelerator. Beam position monitors, buried inside the accelerator tunnel, continuously collect very large amount of position data and transmit them to the data acquisition and analysis system above ground. This dataset is essential to fine-tune the accelerator to produce highest number of collisions.

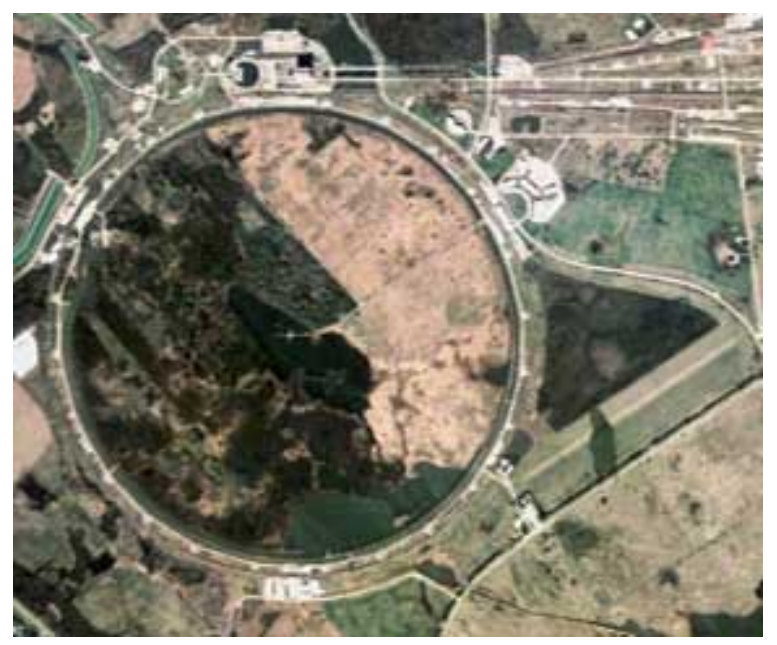

Figure 1: Tevatron accelerator complex

The circular ring in the Figure 1 is the Tevatron accelerator. The existing system consists of 236 beam position monitors (BPMs) around the underground tunnel of the accelerator. Figure 2 shows the sketch of cylindrical BPMs with two cylindrical detecting plates mounted inside. These plates detect the existence of proton and antiprotons. Associated signals are transmitted to the dedicated above ground VME crates through the pickup cables attached to the plates. Each of the 27 service buildings, indicated by small, white structures around the ring, will contain one VME crate and associated instrumentation. These crates are connected to a dedicated communication network for the accelerator complex. When the Tevatron accelerator is operational, signals received from the BPMs are used to perform a number of control, calibration and diagnostic tasks.

The existing system can only capture the proton signals from the accelerator. The new system, when fully operational, will be able to capture combined proton and antiproton signals and will be able to separate the antiproton signal from the combined signal at high resolution. This significant enhancement was beyond the envelope of technical capabilities when the Tevatron was constructed about two decades ago. The upgrade is mainly focused on achieving dramatically better resolution (more bits) and vastly superior reliability using new modern electronics.
The new system is designed to take advantage of exceptional progresses made in the hardware and software technologies in past two decades. The approximate length of the project is sixteen months with a budget of approximately four million dollars with additional contingencies.

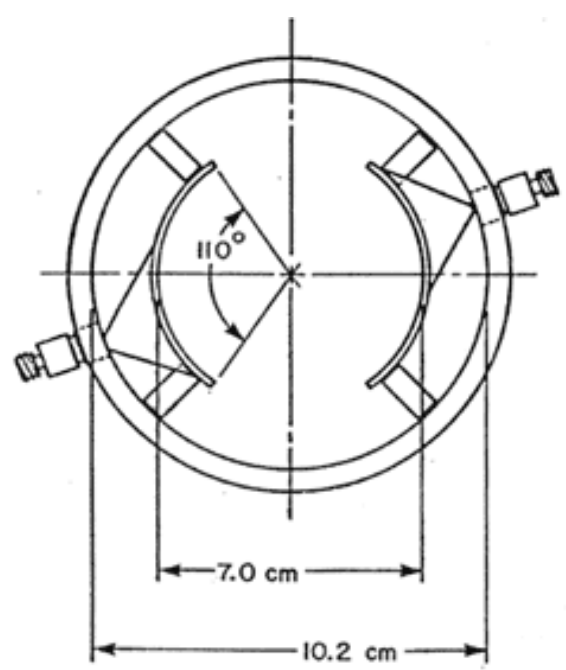

Figure 2: Cartoon of Tevatron BPM

As shown in the figure below, the system has four major subsystems. Electronics subsystem includes $27 \mathrm{VME}$ based hardware units consisting of a timing module, several filter modules, and several special purpose Digital Receiver boards. The system is programmable using firmware developed in-house. Almost all modules of this system are custom made. Front-end software includes VxWorks based data acquisition system. It performs preliminary processing that communicates to the accelerator control and other systems. This subsystem is developed using modern methodologies and will be easily extendable and maintainable. The online software system keeps track of the communication and numerous system parameters. The online component of the project is mostly enhancement of the existing software although various library routines will be enhanced. This subsystem requires the least amount of work. Offline software, on the other hand, requires significant amount of work, particularly in the areas of new software for proton and anti-proton separation and understanding the nature of the signals.

\section{Project risks}

Since the initial installation of the BPM system was done about twenty years ago, only minimal project documentation of the system is available. No usable cost and schedule information was available to the project 
team to use as a baseline. Consequently, the project is treated as a new project using standard development practices. Like other projects, the Tevatron BPM project is constrained by the cost and schedules complicated by project specific caveats.
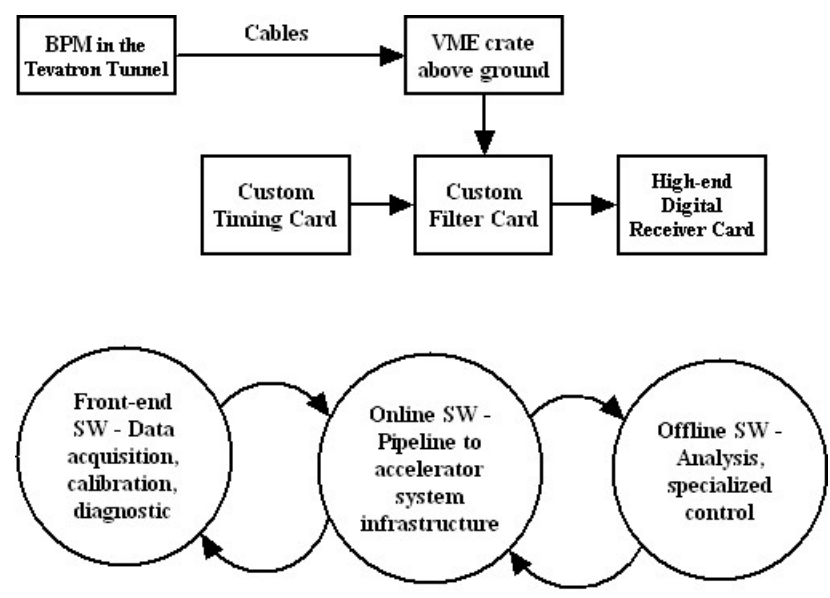

Figure 3: BPM Subsystems

From early on, it was important to estimate, track, and report the cost and schedule accurately. The schedule is tightly driven by the accessibility to the Tevatron accelerator service buildings. When the accelerator is in operation, engineers could take crucial test data to develop algorithms. However, any integration testing or installation can only be done during a specific window of three months when the accelerator is not operating. The hardware must be fabricated before the installation in the service buildings during this window of time. The window of opportunity, if missed, can impact the system delivery schedule critically. To meet the demand of scientists, the newly installed BPM system must perform according to the established requirements after a reasonable commissioning period. Any schedule slip in early phases of the project will affect the final outcome of the project significantly.

Apart from the cost and schedule risks, there are two major risks associated with scientific projects. First one, defined as the risk of discovery in this paper, is the risk that the system proposed may not be entirely feasible with the capabilities of the modern hardware or firmware, even though early simulations/emulations indicate general feasibility. Since there is often no precedence for scientific systems proposed, such a risk has to be managed continuously throughout the project. The second risk, risk to scientific investment, is equally important. If the commissioned system meets, or preferably exceeds, expectations of the scientific community, scientific careers and prestige of the laboratory are enhanced.
Decent metrics to measure such intangible qualities are not yet available. Standard industrial systems must be economically feasible and only incremental improvements on existing systems are attempted. However, improvements for scientific systems often demand designs that are beyond the envelope of existing knowledge. The risk of discovery of such designs poses immediate risk to availability of the scientific facility to the users. The cost of discovery associated with the scientific systems, that must push the envelope, is not fully understood yet. Although the scientific system development process is not different from the standard industrial system development process, they are different during the system design phase. For scientific systems, it is important to understand the design well.

\section{Methodology}

The initial plan for the project was modeled after a generic BPM system already implemented in another accelerator. However, toward the end of the requirements phase, scientists and engineers realized that the new BPM system is more complex than initially understood. The design challenges became more complex than it was originally envisioned. However, in our environment, the fund must be estimated and allocated before the full design can be fleshed out. Consequently, the initial estimate for the labor and equipment, based on the existing system, had to be modified significantly. The investigation for possible technical choices involved careful analysis of proton specific data collected using an older version of the Digital Receiver board, generic filter and Tevatron under full-scale operation. New algorithms developed using the analysis of real data and simulations was presented to a group of accelerator experts during a Technical Choice Review. The design process for the project continued to evolve as designers discovered technical caveats and complexities of the system.

Although development of the requirements for the system needed a significant amount of understanding, system users knew what they wanted from the enhanced system. However, it was clear that the design process must accommodate a continuous process of discovery. Variations in the project cost due to discovery process, termed as discovery cost, can provide us with some clues about the nature of scientific projects.

As shown in the next figure, the project WBS is composed of five major sections. The first four of them represent based on the system life-cycle phases, namely, design, fabrication, installation and commissioning. The project management section, that embraces work for all phases, includes project management support and technical coordination activities. The MS project 2002 project management tool used for the Tevatron BPM 
project allowed us to calculate and monitor variables associated with the project earned values throughout the system life cycle. Tracking tools and earn value measures provided by the tool is used extensively by the project. As shown later, Estimate at Completion (EAC) data gathered for the design phase seemed to be most revealing indicator of the dynamics of the design. EAC for a particular task is defined to be the sum of costs actually incurred up to the status date and cost estimated for remaining work to be done for a task. This particular design of the project WBS allows us to isolate the costs of components into well-defined system life-cycle phases. At the time of writing the project is at the end of the design phase.

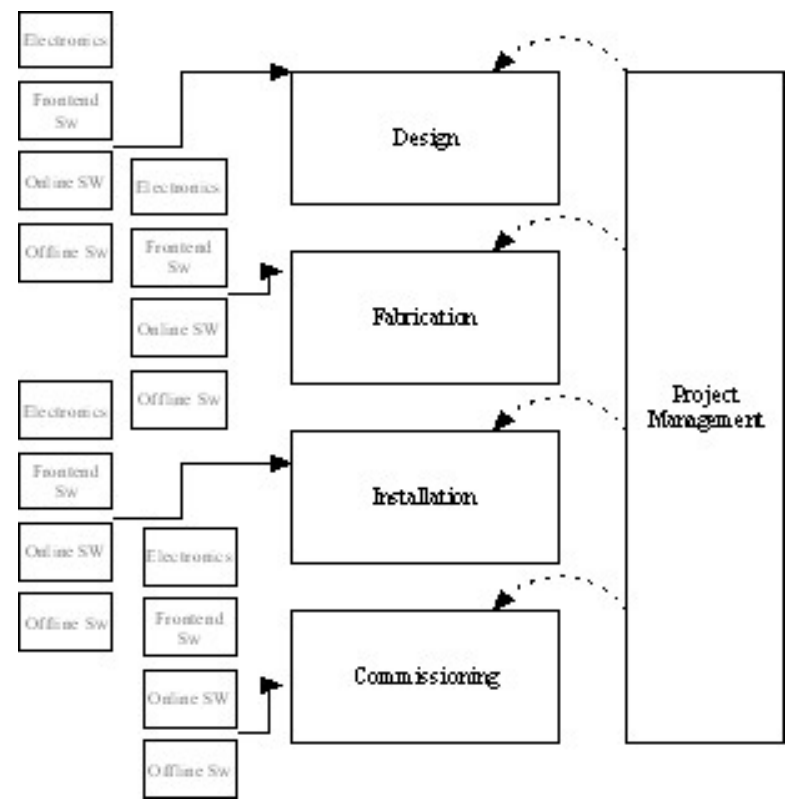

Figure 4: Phase Dependant WBS organization

To quantify the cost of discovery process, the time evolution study of various earned value variables is known to be most beneficial. However, it is often difficult to collect detailed usable data. In the Tevatron BPM project, it was possible to collect adequate earned value data. The easiest data to collect was the basic estimated cost at various stages of the project.

More interestingly, we could also collect reliable EAC data for the design phase of the system development. Although evolution of other earned value variables provide important sanity checks for the project other variables, EAC proved to be best metric for our purpose. EAC depends on the project baselines and status of the project at a particular date. Since the phases and subsystems in the WBS are isolated from each other, it is only necessary to wait for the end of the design phase to obtain a good understanding of the role of discovery in the design. It is not necessary to wait for the end of the project. Some results of early work for fabrication phase are available. As expected, EAC values are significantly more stable. The following two figures show two different views of the EAC data collected from the beginning of the project to the near end of the design phase project.

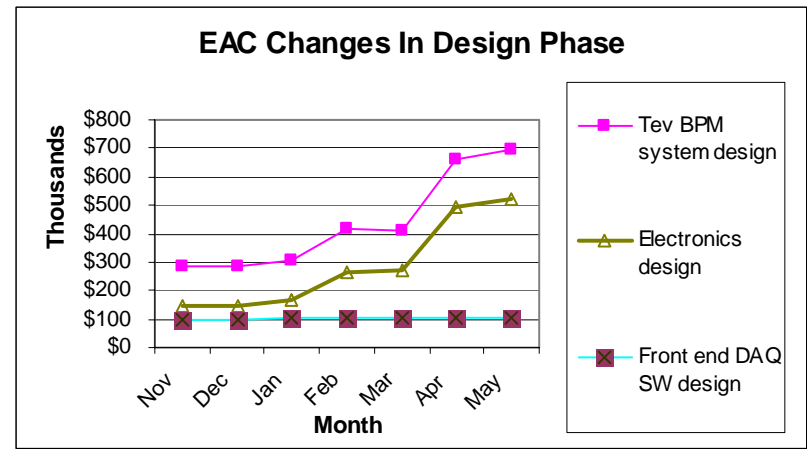

Figure 5: Influence of Discovery Risk on Design

The above figure shows the total EAC for the Tevatron BPM project, EAC for electronic design and front end software design. It should be noted that these values do not include any material purchase cost. The EAC for the electronic design increased most significantly. The first baseline estimate for the project was done using proven techniques of project management, including interviews with subject matter experts and detailed work estimation. The cost increases for this component in the design phase is due to of larger than expected design effort for the enhancements to the hardware. The cost increases coincides with the spike in design change efforts. Consequently, the excess cost can be attributed to the cost of discovery. Major enhancements to the new hardware system involved:

- Enhanced digital receiver boards significantly modified by vendor. Cost of these boards is the most significant portion of total cost of the project. Consequently, design phase included detailed cost/benefit analysis to decide on make or buy decisions, bidding process, prototype approval, and long production lead time. Significant amount of simulation work was also necessary

- Custom analog/anti-aliasing filter by vendor. Defining the filter specification with significantly tight tolerances was a significant effort. Finding an adequate vendor involved significant cost as well.

In contrast with the electronic design, the EAC plot for the front end software indicates the stability of the design 
phase. The VxWorks based data acquisition software is well-understood by the team. Experts from other areas provided accurate interface information.

Using EAC variables, the figure below shows the comparison of front end software with the online software and offline software. Like front end software, online software is well-understood. On the other hand, offline software EAC values increase for offline software. This component of the Tevatron software involves understanding completely new algorithms for the signal logic and the separation of proton and antiproton signals.

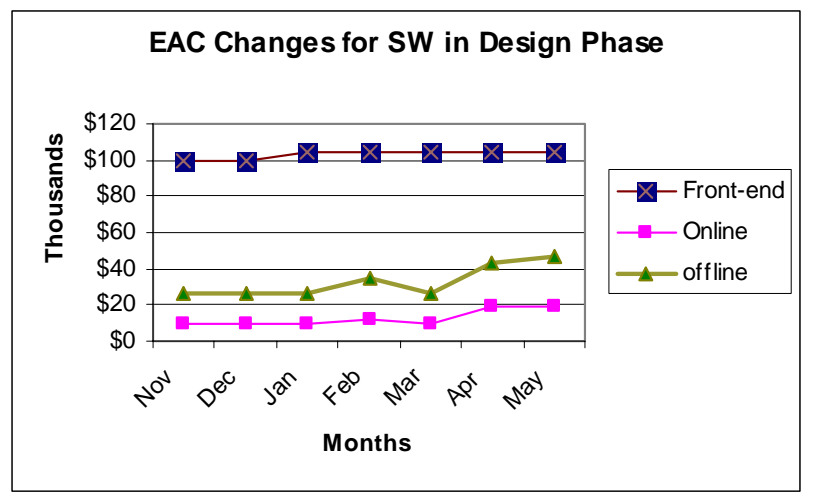

Figure 6: Influence of Discovery on Software Design

\section{Conclusion}

The EAC profiles remained relatively steady for the first three months of the project, indicating that the estimation worked initially. Significant increases in the EAC for electronic design and offline software can only be attributed to the cost of innovations. This profile can be used to distribute work for later phases, that is, fabrication, installation and commissioning. It should also be noted that the cost of discovery also depends on scientific and associated business environments. Availability of similar historical profiles of phase based EAC variables for scientific projects in similar environment can be extremely valuable for future project cost estimation.

\section{Acknowledgement}

The author wishes to acknowledge the support of her supervisor Dr. Stephen Wolbers and Fermi National Accelerator Laboratory, operated by Universities Research Association Inc. under Contract No. DE-AC0276CH03000 with the United States Department of Energy.

\section{References}

[1] A guide to the Project Management Body of Knowledge (Adoption of PMI Standard), IEEE Standard 1490-1998, IEEE, 1998

[2] EIA Standard EIA-748-A, "Earned Value Management Systems”, January 2002.

[3] Tennenhouse, David, "Innovation breeds Success at Intel," IEE Engineering Management, December/January 2003/04 